\title{
Comparison of Percutaneous Mesh-Container- Plasty and Pedicle Screw Fixation Plus Vertebroplasty for Thoracolumbar Osteoporotic Vertebral Burst Fractures: A Retrospective Study
}

\section{Chengxuan Tang}

Third affiliated Hospital of Wenzhou Medical University

\section{Xiaojun Tang}

Third affiliated Hospital of Wenzhou Medical University

\section{Yimin Li}

Third affiliated Hospital of Wenzhou Medical University

\section{Xiqiang Zhong}

Wenzhou Medical University Second Affiliated Hospital

\section{Weihao Zhang}

Third affiliated Hospital of Wenzhou Medical University

Shaoqi He ( $\square$ heshaoqi@126.com )

Third affiliated Hospital of Wenzhou Medical University https://orcid.org/0000-0002-9368-2228

\section{Research article}

Keywords: Spinal fracture, pedicle screw fixation, mesh-container, osteoporosis, vertebroplasty.

Posted Date: September 28th, 2020

DOI: https://doi.org/10.21203/rs.3.rs-76171/v1

License: (c) (i) This work is licensed under a Creative Commons Attribution 4.0 International License.

Read Full License 


\section{Abstract}

Background: Percutaneous mesh-container-plasty (PMCP) and pedicle screw fixation plus vertebroplasty (PSFV) were developed with advantages of bone cement leakage, height restoration and kyphotic angle reduction in osteoporotic vertebral compression fractures (OVBF). The purpose of the current study was to compare the effects and safety between PMCP and PSFV for treatment of thoracolumbar OVBF.

Methods: A retrospective study of 227 consecutive patients with thoracolumbar OVBF without neurologic deficit who underwent PMCP $(n=109)$ or PSFV $(n=118)$ was conducted. The epidemiological data, surgical outcomes, clinical and radiological features were compared between the 2 groups. Clinical evaluation of VAS and ODI, and radiological evaluation of height restoration, deformity correction, cement leakages and canal compromise were calculated on preoperative, postoperative and 2 years postoperative.

Results: Cost( $4.82 \pm 0.21$ vs. $5.50 \pm 0.29, P<0.05)$, blood loss(7.36 \pm 3.67 vs. $22.52 \pm 4.79, P<0.05)$, operation time(34.35 \pm 8.72 vs. $92.70 \pm 17.24, P<0.05)$ and hospital day(4.42 \pm 1.62 vs. $5.46 \pm 2.31, P<0.05)$ were significantly lower in the PMCP group than in the PSFV group. VAS $[2(1,3) v s .3(2.75,4), P<0.05]$ and ODI $(21.01 \pm 7.90$ vs. $33.81 \pm 8.81, P<0.05)$ scores were significantly lower in the PMCP group than in the PSFV group postoperative. However, no statistical difference was found in VAS and ODI between group PMCP and PSFV 2 years postoperative. Anterior( $85.63 \pm 8.22 \%$ vs. $74.86 \pm 12.60 \% ; 83.49 \pm 8.95 \%$ vs. $68.77 \pm 11.32 \% ; P<0.05)$ and middle vertebral body height ratio(84.68 $\pm 7.69 \%$ vs. $71.05 \pm 12.68 \%$; $82.47 \pm 8.34 \%$ vs. $74.96 \pm 12.90 \% ; P<0.05)$ were significantly higher in the PSFV group than in the PMCP group postoperative. Cobb angle( $7.95 \pm 4.42$ vs. $11.99 \pm 5.69,8.59 \pm 4.46$ vs. $12.95 \pm 5.74 ; P<0.05)$ and canal compromise(19.85 \pm 6.18 vs. $10.18 \pm 6.99,10.03 \pm 7.20$ vs. $19.90 \pm 5.98 ; P<0.05)$ were significantly lower in group PSFV than in the PMCP group postoperative. No statistical difference was found in cement leakage between group PMCP and PSFV.

Conclusion: Despite of relative worse radiological results, PMCP is a safe, minimally invasive, and costeffective surgical method and can obtain better satisfactory clinical results compared to PSFV for OVBF.

\section{Background}

Owing to the demographic shift towards an older society, annual incidence of osteoporosis and its associated fractures are seen increasingly in the world. Osteoporotic vertebral fractures (OVF) can affect the patient's quality of life, including chronic back pain, functional limitations, depression and disability, which have risen to be a major health problem ${ }^{[1]}$.

Thoracolumbar osteoporotic vertebral burst fractures (OVBF) are severe type of OVF. The management of OVBF has not been properly coded to date. However, surgical treatment of these fractures seems to reduce pain and mobilize the patients more quickly, and the hospital day is therefore shorter in this case. Many patients with OVBF without neurologic deficit have recently undergone kyphoplasty with good clinical and radiological results ${ }^{[2-4]}$. However, there have been some complications including cement 
leakage, loss of the restored height and kyphotic alignment after balloon deflation prior to cement injection $^{[5-7]}$.

In order to avoid these complications, percutaneous mesh-container-plasty (PMCP) ${ }^{[8]}$ and pedicle screw fixation plus vertebroplasty (PSFV) ${ }^{[9,10]}$ were developed with advantages of cement leakage, height restoration and kyphotic angle reduction. During the cement injection process, the continuous cement injection makes the mesh container produce a pressure, then cement leaks outside of the mesh container and enters the bone trabeculae. Thereby, better inhibition ability of cement leakage can be reached. The mesh container remains within the newly created vertebral cavity so the balloon can be removed after deflation while preventing the vertebral body from collapsing. So that, virtually physiological vertebral body height and shape might be restored and preserved. Pedicle screw fixation reduced the fracture by ligamentotaxis before performing vertebroplasty and decreased the risk of cement leakage.

Based on these previous studies, we hypothesize that there will be differences in the clinical efficacy and safety of PMCP and PSFV for the treatment of OVBF. To test our hypothesis, this study will thus compare the clinical and radiological results of PMCP and PSFV for the treatment of OVBF.

\section{Methods}

\section{Study design}

Ethical approval for this retrospective study was provided by the Ethics Committee of the authors' institute. We routinely obtain written informed consent for accumulation of clinical data for future retrospective analyses from each patient who receives PMCP or PSFV at our hospital, including all patients in this study. The medical records of consecutive patients sustained OVBF without neurologic deficit and underwent PMCP or PSFV from May 2015 to April 2018 were reviewed (Fig. 1).

First, we selected 253 patients who received PMCP or PSFV. Inclusion criteria were as follows: 1) elderly ( $\geq 60$ years), 2) thoracolumbar(T10 to L2) single fresh complete burst fracture (type A3 or A4 according to AOSpine thoracolumbar spine injury classification system $)^{[11]}, 3$ ) without neurological deficit, 4) being diagnosed with osteoporosis according to $T$ value of dual-energy $x$-ray absorptiometry (DXA) less than -2.5. We then excluded poly-traumatized and other OVF types patients, and patients with symptoms of neurological deficits, preexisting spinal deformity or previous spinal operation, metastatic bone tumor or multiple myeloma, systemic or local infections and severe bleeding disorders. Finally, we analyzed 227 patients who were divided into two groups according to surgical techniques-PMCP $(n=109)$ and PSFV $(n=118)$ groups.

Preoperatively, standard clinical examination and evaluation including of the medical history, physical examination of percussion pain, assessment of the pain intensity [visual pain analogue scale (VAS)] , and the activity level [Oswestry Disability Index (ODI)] ${ }^{[12]}$ were evaluated. X-rays of the relevant spinal region in two planes, Computed Tomography (CT) scan and magnetic resonance imaging (MRI) (T1-weighted 
and T2-weighted sequences including short tau inversion recovery sequences) and DXA were performed. All patients received a calcium supplementation (1000 mg of elemental calcium daily), and vitamin D (400 600 UI of vitamin D). Twenty patients in group PMCP and 27 patients in group PSFV received hormonal replacement therapy (estrogens and progestin). Bisphosphonates were given to 89 patients (zoledronate, $\mathrm{n}=25$; alendronate, $\mathrm{n}=64$ ) in group PMCP and 91 patients in group PSFV (zoledronate, $\mathrm{n}=$ 33; alendronate, $n=58$ ).

\section{Surgical technique}

In PSFV group, all surgical procedures were performed under general anesthesia with endotracheal intubation. Patients were placed in a prone position on four bolsters placed on a radiolucent operating table with the abdomen freely suspended. Patients were installed with surgical bolsters placed under the thorax and the iliac crests in order to induce spinal lordosis and facilitate the reduction of the fracture. The manual lordotic manoeuvre was first performed to correct kyphosis. Percutaneous pedicle screw fixation was performed using Zina ${ }^{\mathrm{TM}}$ device (Sanyou Medical Co., Ltd, Shanghai, China) under frontal and lateral scopic control. A targeting cannulated needle (Sanyou Medical Co., Ltd, Shanghai, China) for each pedicle of the instrumented adjacent vertebrae is used to locate the pedicle. K-wires of $2 \mathrm{~mm}$ are then passed through the needle.After removal of the targeting needle, cannulated pedicle screws were placed with extender sleeves in down into the pedicles of the non fractured vertebrae above and below the fractured vertebra and subsequently removal of all $4 \mathrm{~K}$-wires. Lordotic manoeuvre was performed to restore vertebral body height and correct kyphosis. Following a 6-mm diameter trocar (Dragon Crown Medicial Co., Ltd., Jinan City, Shandong Province, China) in a cannula was inserted into opposite pedicle at the fractured vertebra. The position was controlled by an image intensifier, which was then enlarged with the use of an access cannula with a trocar. Once the cannula reached the optimal position, after removing the trocar, Polymethylmethacrylate (PMMA) cement was injected into the defect of the fractured body through the cannula under continuous fluoroscopic monitoring $\triangle$ Figure $1 \otimes$. The PMMA insertion was considered complete when it reached the posterior third of the vertebral body.

In PMCP group, all surgical procedures were performed under local anesthesia. Patients were positioned in a prone position on four bolsters placed on a radiolucent operating table with the abdomen freely suspended. A $1 \mathrm{~cm}$ skin incision was made lateral to the desired entry point of the pedicle to percutaneously. A trocar (Dragon Crown Medicial Co., Ltd., Jinan City, Shandong Province, China) in a cannula was inserted into pedicle at the fractured vertebra through pedicular approach as a working channel. After removing the trocar, a balloon was placed into the working channel and slowly inflated to create a low-pressure cavity for cement injection. Inflation continued until the balloon pressure up to 300 psi. Antero-posterior radiograph shows the balloon exceed the midline of the vertebra. Then the balloon was deflated and removed. If the balloon does not exceed the midline of the vertebra, a bilateral puncture is required. Then the balloon was deflated and removed. a mesh container (Dragon Crown Medicial Co., Ltd., Jinan City, Shandong Province, China) was advanced into the cavity. The mesh container is made of polyethylene terephthalate (PET). Then PMMA cement was manually injected into the mesh container within the treated vertebral body by applying a cement perfusion apparatus under fluoroscopic guidance. 
With the continuous injection of PMMA, the mesh container was inflated and the height of the fractured vertebral was restored. At a certain injection amount, PMMA cement leaked outside the mesh container from the meshes and entered bone trabeculae.The PMMA insertion was considered complete when it reached the posterior third of the vertebral body or had a potential tendency of cortical, epidural, and anterior venous cement leakage(Figure 2).

Operation time, estimated blood loss and PMMA volume were recorded. All patients were postoperatively followed clinically and radiographically the day after surgery, three months, six months and at every oneyear interval thereafter. Patients were assessed for any neurologic complications. As for CT scan, all patients had it after surgery, one year and two years later. Segmental kyphosis, anterior and middle vertebral body height ratio (AVBHr and $\mathrm{MVBHr}$ ), and canal compromise were measured using lateral radiograph and CT images. Cement leakage was determined using CT images of all section of fractured vertebra. Back pain intensity was recorded on VAS. Functional outcome was measured using the ODI. Three independent blinded spine surgeons finished the clinical evaluation of patients. Additionally, two other independent blinded spine surgeons assessed the radiographs.

\section{Statistical analysis}

SPSS 18 was used for statistical analysis. Continuous variables are expressed as mean \pm standard deviation. Statistical analysis was performed with for changes of each radiographic and functional parameter. Independent data, including age, body mass index(BMI), T-score, injury time, operation time, blood loss, hospital day, cost, and injected cement volume, were compared between groups PMCP and PSFV using the student $t$ test. Differences in gender, distribution of fractured vertebral, BMI and cement leakages between two groups were compared using the chi-square test. Repeated measure ANOVA was used to compare VAS, ODI, AVBHr, MVBHr, canal compromise and Cobb angle between two groups. Statistically significant differences were defined at a $95 \%$ confidence level.

\section{Results}

The clinical characteristics of the 227 patients were summarized in Table 1. There were no statistical differences in demographic data including age, gender, distribution of fractured vertebral, T-score, BMI and injury time between the 2 groups $(P>0.05)$. The mean operation time( $34.35 \pm 8.72$ vs. $92.70 \pm 17.24$, $P<0.05)$, blood loss(7.36 \pm 3.67 vs. $22.52 \pm 4.79, P<0.05)$, hospital day $(4.42 \pm 1.62$ vs. $5.46 \pm 2.31, P<$ $0.05)$ and cost $(4.82 \pm 0.21$ vs. $5.50 \pm 0.29, P<0.05)$ were significantly lower in the in PMCP group than in the PSFV group. There were no statistical differences in cement volume between the 2 groups. The details are shown in Table 2. 
Table 1

Basic characteristics and comparative analysis between PMCP and PSFV for the treatment of the 227 patients with thoracolumbar OVBF in this study $(\bar{X} \pm s)$.

\begin{tabular}{|c|c|c|c|c|}
\hline & $\mathrm{PMCP}(n=109)$ & $\operatorname{PSFV}(n=118)$ & $t\left(x^{2}\right)$ & $P$ \\
\hline Age (y) & $73.29 \pm 8.07$ & $72.72 \pm 8.03$ & 0.536 & 0.592 \\
\hline Male/Female & $34 / 75$ & $39 / 79$ & 0.090 & 0.778 \\
\hline Distribution & & & 4.972 & 0.290 \\
\hline T10 & 26 & 27 & & \\
\hline T11 & 27 & 22 & & \\
\hline T12 & 17 & 31 & & \\
\hline L1 & 24 & 17 & & \\
\hline L2 & 21 & 21 & & \\
\hline \multicolumn{5}{|l|}{ Fracture type } \\
\hline A3 & 63 & 67 & \multirow[t]{2}{*}{0.024} & \multirow[t]{2}{*}{0.894} \\
\hline A4 & 46 & 51 & & \\
\hline T-score & $-3.10 \pm 0.44$ & $-3.17 \pm 0.48$ & 1.098 & 0.273 \\
\hline BMI & $23.22 \pm 4.20$ & $23.14 \pm 4.09$ & 0.150 & 0.881 \\
\hline Injury time (d) & $4.18 \pm 2.09$ & $4.05 \pm 1.85$ & 0.507 & 0.613 \\
\hline
\end{tabular}


Table 2

Patient's perioperative parameters comparison between group PMCP and PSFV for the treatment of the 227 patients with thoracolumbar OVBF in this study $(\bar{X} \pm s)$.

\begin{tabular}{|lllll|}
\hline & PMCP(n=109) & PSFV( $n=118)$ & $t\left(\chi^{2} / Z\right)$ & $P$ \\
\hline Operation time(min) & $34.35 \pm 8.72$ & $92.70 \pm 17.24$ & $t=-32.186$ & $<0.001$ \\
\hline Blood loss(ml) & $7.36 \pm 3.67$ & $22.52 \pm 4.79$ & $t=-26.888$ & $<0.001$ \\
\hline Hospital day(d) & $4.42 \pm 1.62$ & $5.46 \pm 2.31$ & $t=-3.934$ & $<0.001$ \\
\hline Cost(thousand dollar) & $4.82 \pm 0.21$ & $5.50 \pm 0.29$ & $t=-20.183$ & $<0.001$ \\
\hline Cement leakages & $19 / 109$ & $22 / 118$ & $\chi^{2}=0.056$ & 0.864 \\
\hline Cement volume(ml) & $6.19 \pm 1.74$ & $6.22 \pm 1.96$ & $t=-0.112$ & 0.911 \\
\hline $\begin{array}{l}\text { PMCP: Percutaneous mesh-container-plasty; PSFV: Pedicle screw fixation plus vertebroplasty; OVBF: } \\
\text { Osteoporotic vertebral burst fractures. }\end{array}$ & & & \\
\hline
\end{tabular}

\section{Clinical Evaluation}

VAS scores were reduced from preoperative $7(6,8)$ to postoperative $2(1,3)(P<0.05)$ and $2(1,2) 2$ yeas postoperative in group PMCP, and from preoperative $7(6,8)$ to postoperative $3(2.75,4)$ and $1(1,2.25) 2$ yeas postoperative in group PSFV $(P<0.05)$. ODI scores were improved from preoperative $67.26 \pm 12.79$ to postoperative $21.01 \pm 7.90$ and $16.40 \pm 7.292$ yeas postoperative in group PMCP $(P<0.05)$, and from preoperative $67.36 \pm 13.11$ to postoperative $33.81 \pm 8.81$ and $15.47 \pm 7.652$ yeas postoperative in group PSFV $(P<0.05)$. Moreover, VAS and ODI scores were significantly lower in the PMCP group than in the PSFV group postoperative $(P<0.05)$. However, there was no difference in VAS score between the PMCP and PSFV groups 2 years postoperative $(P>0.05)$. The details are shown in Table 3. 
Table 3

Clinical comparisons between group PMCP and PSFV for the treatment of the 227 patients with thoracolumbar OVBF in this study $(\bar{X} \pm \mathrm{s}$ or Median $(\mathrm{QR}))$.

\begin{tabular}{|c|c|c|c|c|}
\hline & $\operatorname{PMCP}(n=109)$ & $\operatorname{PSFV}(n=117)$ & $t(Z)$ & $P$ \\
\hline \multicolumn{5}{|l|}{ VAS } \\
\hline Preoperative & $7(6,8)$ & $7(6,8)$ & $Z=-0.516$ & 0.606 \\
\hline Postoperative & $2(1,3)^{\star}$ & $3(2.75,4) \star$ & $Z=-6.716$ & $<0.001$ \\
\hline 2 years postoperative & $2(1,2)^{\star}$ & $1(1,2.25)^{\star}$ & $Z=-0.288$ & 0.774 \\
\hline \multicolumn{5}{|l|}{ ODI } \\
\hline Preoperative & $67.26 \pm 12.79$ & $67.36 \pm 13.11$ & $t=-0.058$ & 0.954 \\
\hline Postoperative & $21.01 \pm 7.90 *$ & $33.81 \pm 8.81^{*}$ & $t=-11.486$ & $<0.001$ \\
\hline 2 years postoperative & $16.40 \pm 7.29 *$ & $15.47 \pm 7.65^{\star}$ & $t=0.937$ & 0.350 \\
\hline \multicolumn{5}{|c|}{$\begin{array}{l}\text { * Repeated measures variance analysis was used for the statistical analysis. There were significant } \\
\text { differences }(P<0.05) \text { between postoperative or } 2 \text { years postoperative and preoperative of these two } \\
\text { groups. PMCP: Percutaneous mesh-container-plasty; PSFV: Pedicle screw fixation plus vertebroplasty; } \\
\text { OVBF: Osteoporotic vertebral burst fractures; VAS: Visual pain analogue scale; ODI: Oswestry } \\
\text { Disability Index. }\end{array}$} \\
\hline
\end{tabular}

Therefore, PMCP treatments had significant rapid ability in pain relief and functional recovery than PSFV treatments.

\section{Radiologic Evaluation}

AVBHr, MVBHr and Cobb angle scores were improved from preoperative $(64.88 \pm 11.02 \%, 71.00 \pm 12.57 \%$, $\left.13.51 \pm 5.64^{\circ}\right)$ to postoperative $\left(74.86 \pm 12.60 \%, 74.89 \pm 12.76 \%, 11.99 \pm 5.69^{\circ}\right)$ and $(68.77 \pm 11.32 \%$, $\left.71.04 \pm 12.56 \%, 12.95 \pm 5.74^{\circ}\right) 2$ years postoperative in group PMCP $(P<0.05)$, and from preoperative $\left(64.60 \pm 9.02 \%, 70.81 \pm 7.88 \%, 13.44 \pm 5.07^{\circ}\right)$ to postoperative $\left(85.63 \pm 8.22 \%, 84.68 \pm 7.69,7.95 \pm 4.42^{\circ}\right)$ and $\left(83.49 \pm 8.95 \%, 82.28 \pm 8.23 \%, 8.59 \pm 4.46^{\circ}\right) 2$ years postoperative in group PSFV $(P<0.05)$. Canal compromise were improved from preoperative $(20.46 \pm 7.48)$ to postoperative $(10.18 \pm 6.99)$ and $(10.03 \pm$ 7.20) 2 years postoperative in group PSFV $(P<0.05)$. There was no difference in canal compromise between preoperative, postoperative and 2 years postoperative in group $\operatorname{PMCP}(P>0.05)$. Moreover, $\mathrm{AVBHr}$, $\mathrm{MVBHr}$, canal compromise and Cobb angle scores were were significantly lower in the PMCP group than in PSFV group postoperative and 2 years postoperative $(P>0.05)$. All radiographic results are shown in Table 4. 
Table 4

Radiologic comparisons between group PMCP and PSFV for the treatment of the 227 patients with thoracolumbar OVBF in this study $(\bar{\chi} \pm s)$.

\begin{tabular}{|c|c|c|c|c|}
\hline & $\operatorname{PMCP}(n=109)$ & PSFV(n = 117) & $t$ & $P$ \\
\hline \multicolumn{5}{|l|}{ AVBHr (\%) } \\
\hline Preoperative & $64.88 \pm 11.02$ & $64.60 \pm 9.02$ & 0.205 & 0.873 \\
\hline Postoperative & $74.86 \pm 12.60 *$ & $85.63 \pm 8.22 *$ & -7.560 & $<0.001$ \\
\hline 2 years postoperative & $68.77 \pm 11.32^{\star}$ & $83.49 \pm 8.95^{\star}$ & -10.810 & $<0.001$ \\
\hline \multicolumn{5}{|l|}{ MVBHr (\%) } \\
\hline Preoperative & $71.00 \pm 12.57$ & $70.81 \pm 7.88$ & 0.136 & 0.892 \\
\hline Postoperative & $74.89 \pm 12.76^{\star}$ & $84.68 \pm 7.69 *$ & -6.932 & $<0.001$ \\
\hline 2 years postoperative & $71.04 \pm 12.56^{\star}$ & $82.28 \pm 8.23^{\star}$ & -7.906 & $<0.001$ \\
\hline \multicolumn{5}{|l|}{ Cobb angle $\left(^{\circ}\right)$} \\
\hline Preoperative & $13.51 \pm 5.64$ & $13.44 \pm 5.07$ & 0.105 & 0.916 \\
\hline Postoperative & $11.99 \pm 5.69 *$ & $7.95 \pm 4.42^{\star}$ & 5.942 & $<0.001$ \\
\hline 2 years postoperative & $12.95 \pm 5.74^{\star}$ & $8.59 \pm 4.46^{\star}$ & 6.355 & $<0.001$ \\
\hline \multicolumn{5}{|l|}{ Canal compromise (\%) } \\
\hline Preoperative & $20.12 \pm 5.72$ & $20.46 \pm 7.48$ & -0.393 & 0.695 \\
\hline Postoperative & $19.85 \pm 6.18$ & $10.18 \pm 6.99 *$ & 11.007 & $<0.001$ \\
\hline 2 years postoperative & $19.90 \pm 5.98$ & $10.03 \pm 7.20 *$ & 11.255 & $<0.001$ \\
\hline \multicolumn{5}{|c|}{$\begin{array}{l}\text { * Repeated measures variance analysis was used for the statistical analysis. There were significant } \\
\text { differences }(P<0.05) \text { between postoperative or } 2 \text { years postoperative and preoperative of these two } \\
\text { groups. PMCP: Percutaneous mesh-container-plasty; PSFV: Percutaneous pedicle screw fixation plus } \\
\text { vertebroplasty; OVBF: Osteoporotic vertebral burst fractures; AVBHr: Anterior vertebral body height } \\
\text { ratio; MVBHr: Middle vertebral body height ratio. }\end{array}$} \\
\hline
\end{tabular}

Therefore, PSFV group had significantly higher height restoration, improvement in segmental kyphosis and canal compromise than PMCP group.

\section{Surgical Complications}

Cement leakage was observed in 17.43\%(19/109) cases of PMCP group (ten into the disc or around vertebral body through a cortical defect, 6 into segmental vein, and 3 into spinal canal via the basivertebral vein) and 18.64\% (22/118) of patients had PMMA leakage in PSFV groups (thirteen into the disc or around vertebral body through a cortical defect, 5 into segmental vein, and 4 into spinal canal via the basivertebral vein), respectively $(P>0.05)$ (Table 2). All cement leakage were asymptomatic, and no 
surgical intervention was required to remove the extravasated cement. Postoperative complications, such as neurological functional aggravation, hemorrhage, wound healing abnormalities, infection and pulmonary embolism, failure of posterior instrumentation were not observed during the 2 years follow-up period.

Depending on above analyses, PMCP treatment had similar safety with PSFV treatments for OVBF.

\section{Discussion}

Osteoporosis and associated fractures are prevalent in clinics, especially in women above 50 years old. A standardized treatment strategy for osteoporotic thoracolumbar burst fracture does not exist nowadays ${ }^{[2,}$ 10,13-15]. Percutaneous kyphoplasty(PKP) has been increasingly used in older people currently due to minimally invasive treatments. However, a major disadvantage of PKP is cement leakage, loss of the restored height and kyphotic alignment after balloon deflation prior to cement injection ${ }^{[16,17]}$. Therefore, pedicle screw fixation combined with vertebroplasty and mesh container were developed for the advantages of safety in cement leakage, height restoration and improvement in segmental kyphosis ${ }^{[8,10]}$.

Patients with osteoporosis often present multiple medical comorbidities and poorly endure open surgeries. Minimal invasive techniques have advantages including reserves back muscles, quick return to daily activities, the disappearance of pain, minimal operative risks and comorbidity, and maintenance of stability ${ }^{[18,19]}$. In our study, the operative time and blood loss and hospital day were significantly lower in the in PMCP group than in the PSFV group. After undergoing this minimally invasive surgery, patients are able to return home quickly. For patients using PMCP as our preceding operative method, the average cost is relatively lower than PSFV group.

In our study, the height restoration, improvement in segmental kyphosis and canal compromise in the PSFV group were all higher than those in the PMCP group. Vertebral body height (AVBHr, MVBHr), segmental kyphosis, and canal compromise were significantly improved postoperatiavely and stable over time, with a minimal loss of correction at 2 years postoperative. Previous studies indicated that PKP was useless for height restoration and improvement in segmental kyphosis. The improvement in segmental kyphosis was attributed to postural reduction with cement strengthening. The most significant factors affecting improvement in segmental kyphosis were the fracture type and cement injected volume ${ }^{[20-22]}$.

Previous study showed that improvement in segmental kyphosis in the PMCP group was higher than those in the PKP group in respect to both A3 and A4 fractures. The possible mechanism for height restoration and kyphosis correlation is the inflation of mesh container. Applying a pedicle-screw fixation is known to maintain the restored vertebral height and involve the risk of secondary kyphosis ${ }^{[10,23]}$. Height restoration and improvement in segmental kyphosis of the treated fractured vertebral body might be an important parameter to evaluate the clinical efficacy for minimally invasive techniques. However, previous studies found no correlation between reconstitution of the vertebral body and the clinical outcome (pain reduction) ${ }^{[24,25]}$. In this study, both PMCP and PSFV treatments had significant ability in 
pain relief and functional recovery postoperatiavely and preserved at 2 years postoperative. However, PMCP obtain better satisfactory clinical results postoperatiavely compared to PSFV for OVBF.

Cementoplasty involves risks of complications, including pulmonary embolism, intradiscal cement leakage, neurological deficit, paraplegia and even death ${ }^{[5,7,26]}$. The risk of cement leaking into the spinal canal is greater when the posterior wall has been damaged, as in the case of our burst fractures ${ }^{[27]}$. burst fractures involving the posterior wall with increased risk of cement leakage into the vertebral canal during the classical vertebroplasty and kyphoplasty interventions. Mesh container in PMCP treatment keeps PMMA cement inside the container and only partial cement leaks outside from the mesh to bone trabecula ${ }^{[8]}$. Reduction of the fracture by ligamentotaxis before performing vertebroplasty might also decrease the risk of cement leakage due to the resulting alignment of cortical bone fragments ${ }^{[10,28]}$. After reducing the fracture using pedicle screw fixation, the reduced fracture can be consolidated by injecting cement as anteriorly as possible and stopped if the cement got close to the posterior aspect of the vertebra or leaked into an extra osseous space, which prevents the cement from entering the spinal canal[ ${ }^{[29]}$. In our study, in $17.43 \%(19 / 109)$ cases of PMCP group (ten into the disc or around vertebral body through a cortical defect, 6 into segmental vein, and 3 into spinal canal via the basivertebral vein) and $18.64 \%(22 / 118)$ of patients had PMMA leakage in PSFV groups (thirteen into the disc or around vertebral body through a cortical defect, 5 into segmental vein, and 4 into spinal canal via the basivertebral vein), respectively $(P>0.05)$ Therefore, PMCP treatment had similar inhibition ability of cement leakage compared with PSFV treatments for OVBF.

The shortcomings of our study lie in the fact that the results were available from the small patient population and the short follow-up study. A further limitation of this study seemed to be its retrospective study. Future studies, a prospective randomized controlled study enrolling more patients through longterm follow-up period are needed to compare the PMCP with PSFV more reliably and objectively.

\section{Conclusion}

Despite of relative worse radiological results, PMCP is a safe, minimally invasive, and cost-effective surgical method and can obtain better satisfactory clinical results compared to PSFV for OVBF.

\section{Abbreviations}

\section{PMCP}

Percutaneous mesh-container-plasty; PSFV:Pedicle screw fixation plus vertebroplasty; OVBF:Osteoporotic vertebral burst fractures; PET:Polyethylene terephthalate; AVBHr:anterior vertebral body height ratio; MVBHr:middle vertebral body height ratio; BMI:Body mass index; PKP:Percutaneous kyphoplasty; VAS:Visual pain analogue scale; ODI:Oswestry Disability Index. OVF:Osteoporotic vertebral fractures; DXA:Dual-energy x-ray absorptiometry; CT:Computed Tomography; MRI:Magnetic resonance imaging; ; PMMA:Polymethylmethacrylate. 


\section{Declarations}

Acknowledgements

Not applicable.

\section{Funding}

Not applicable.

\section{Availability of data and materials}

The patients' data were collected in the Third affiliated Hospital of Wenzhou Medical University.

\section{Authors' contributions}

CXT and SQH designed the study. SQH, XJT, and WHZ collected the data. SQH and XJT were involved in the manuscript writing, literature search, data interpretation, and data monitoring. YML and XQZ were responsible for the data collection and analysis. All authors read and approved the final manuscript.

\section{Ethics approval and consent to participate}

This study was conducted with approval from the Ethics Committee of the Third affiliated Hospital of Wenzhou Medical University(Approval number: YJ2020006). Written informed consent to participate was obtained from all participants.

\section{Consent for publication}

We have obtained consent to publish from the participants.

\section{Competing interests}

We confirm that we have read BioMed Central's guidance on competing interests. The authors declare that they have no competing interests.

\section{References}

1. Fehlings MG, Tetreault L, Nater A, et al The Aging of the Global Population: The Changing Epidemiology of Disease and Spinal Disorders. Neurosurgery. 2015. 77Suppl 4: S1-5.

2. Krüger A, Zettl R, Ziring E, Mann D, Schnabel M, Ruchholtz S. Kyphoplasty for the treatment of incomplete osteoporotic burst fractures. Eur Spine J. 2010. 19(6): 893-900.

3. Gan M, Yang H, Zhou F, et al Kyphoplasty for the treatment of painful osteoporotic thoracolumbar burst fractures. Orthopedics. 2010. 33(2): 88-92. 
4. Hiwatashi A, Westesson PL. Vertebroplasty for osteoporotic fractures with spinal canal compromise. AJNR Am J Neuroradiol. 2007. 28(4): 690-2.

5. Kim YJ, Lee JW, Park KW, et al Pulmonary cement embolism after percutaneous vertebroplasty in osteoporotic vertebral compression fractures: incidence, characteristics, and risk factors. Radiology. 2009. 251(1): 250-9.

6. Niu J, Zhou H, Meng Q, Shi J, Meng B, Yang H. Factors affecting recompression of augmented vertebrae after successful percutaneous balloon kyphoplasty: a retrospective analysis. Acta Radiol. 2015. 56(11): 1380-7.

7. Nieuwenhuijse MJ, Van Erkel AR, Dijkstra PD. Cement leakage in percutaneous vertebroplasty for osteoporotic vertebral compression fractures: identification of risk factors. Spine J. 2011. 11(9): 839 $-48$.

8. Chen C, Li D, Wang Z, Li T, Liu X, Zhong J. Safety and Efficacy Studies of Vertebroplasty, Kyphoplasty, and Mesh-Container-Plasty for the Treatment of Vertebral Compression Fractures: Preliminary Report. PLoS One. 2016. 11(3): e0151492.

9. Verlaan JJ, Somers I, Dhert WJ, Oner FC. Clinical and radiological results 6 years after treatment of traumatic thoracolumbar burst fractures with pedicle screw instrumentation and balloon assisted endplate reduction. Spine J. 2015. 15(6): 1172-8.

10. He S, Lin L, Tang $X$, et al The treatment of osteoporotic thoracolumbar severe burst fractures with short pedicle screw fixation and vertebroplasty. Acta Orthop Belg. 2014. 80(4): 493-500.

11. Vaccaro AR, Oner C, Kepler CK, et al AOSpine thoracolumbar spine injury classification system: fracture description, neurological status, and key modifiers. Spine (Phila Pa 1976). 2013. 38(23): 2028-37.

12. Fairbank JC, Couper J, Davies JB, O'Brien JP. The Oswestry low back pain disability questionnaire. Physiotherapy. 1980. 66(8): 271-3.

13. Ender SA, Eschler A, Ender M, Merk HR, Kayser R. Fracture care using percutaneously applied titanium mesh cages (OsseoFix ${ }^{\circledR}$ ) for unstable osteoporotic thoracolumbar burst fractures is able to reduce cement-associated complications-results after 12 months. J Orthop Surg Res. 2015. 10: 175.

14. Bakhsheshian J, Dahdaleh NS, Fakurnejad S, Scheer JK, Smith ZA. Evidence-based management of traumatic thoracolumbar burst fractures: a systematic review of nonoperative management. Neurosurg Focus. 2014. 37(1): E1.

15. Song X, Wang W, Yan Y, Zuo J, Yao N, Lin H. Clinical effect evaluation of percutaneous vertebroplasty combined with the spinal external fixator for the treatment of osteoporotic compressive fractures with posterior vertebral defect. Eur Spine J. 2014. 23(12): 2711-7.

16. Wang Y, Huang F, Chen L, Ke ZY, Deng ZL. Clinical measurement of intravertebral pressure during vertebroplasty and kyphoplasty. Pain Physician. 2013. 16(4): E411-8.

17. Kamano H, Hiwatashi A, Kobayashi N, et al New vertebral compression fractures after prophylactic vertebroplasty in osteoporotic patients. AJR Am J Roentgenol. 2011. 197(2): 451-6. 
18. Nakano M, Kawaguchi Y, Kimura T, Hirano N. Transpedicular vertebroplasty after intravertebral cavity formation versus conservative treatment for osteoporotic burst fractures. Spine J. 2014. 14(1): 3948.

19. Zaryanov AV, Park DK, Khalil JG, Baker KC, Fischgrund JS. Cement augmentation in vertebral burst fractures. Neurosurg Focus. 2014. 37(1): E5.

20. Xu C, Liu HX, Xu HZ. Analysis of related factors on the deformity correction of balloon kyphoplasty. AJNR Am J Neuroradiol. 2014. 35(1): 202-6.

21. Hiwatashi A, Westesson PL, Yoshiura T, et al Kyphoplasty and vertebroplasty produce the same degree of height restoration. AJNR Am J Neuroradiol. 2009. 30(4): 669 - 73.

22. Kanayama M, Oha F, Iwata A, Hashimoto T. Does balloon kyphoplasty improve the global spinal alignment in osteoporotic vertebral fracture. Int Orthop. 2015. 39(6): 1137-43.

23. Gu YT, Zhu DH, Liu HF, Zhang F, McGuire R. Minimally invasive pedicle screw fixation combined with percutaneous vertebroplasty for preventing secondary fracture after vertebroplasty. J Orthop Surg Res. 2015. 10: 31.

24. Feltes C, Fountas KN, Machinis T, et al Immediate and early postoperative pain relief after kyphoplasty without significant restoration of vertebral body height in acute osteoporotic vertebral fractures. Neurosurg Focus. 2005. 18(3): e5.

25. McKiernan F, Faciszewski T, Jensen R. Does vertebral height restoration achieved at vertebroplasty matter. J Vasc Interv Radiol. 2005. 16(7): 973-9.

26. Hong SJ, Lee S, Yoon JS, Kim JH, Park YK. Analysis of intradiscal cement leakage during percutaneous vertebroplasty: multivariate study of risk factors emphasizing preoperative MR findings. J Neuroradiol. 2014. 41(3): 195-201.

27. Walter J, Haciyakupoglu E, Waschke A, Kalff R, Ewald C. Cement leakage as a possible complication of balloon kyphoplasty-is there a difference between osteoporotic compression fractures (AO type A1) and inburst fractures (AO type A3.1). Acta Neurochir (Wien). 2012. 154(2): 313-9.

28. Uchida K, Nakajima H, Yayama T, et al Vertebroplasty-augmented short-segment posterior fixation of osteoporotic vertebral collapse with neurological deficit in the thoracolumbar spine: comparisons with posterior surgery without vertebroplasty and anterior surgery. J Neurosurg Spine. 2010. 13(5): $612-21$.

29. Girardo M, Cinnella P, Gargiulo G, Viglierchio P, Rava A, Aleotti S. Surgical treatment of osteoporotic thoraco-lumbar compressive fractures: the use of pedicle screw with augmentation PMMA. Eur Spine J. 2017. 26(Suppl 4): 546-551.

\section{Figures}




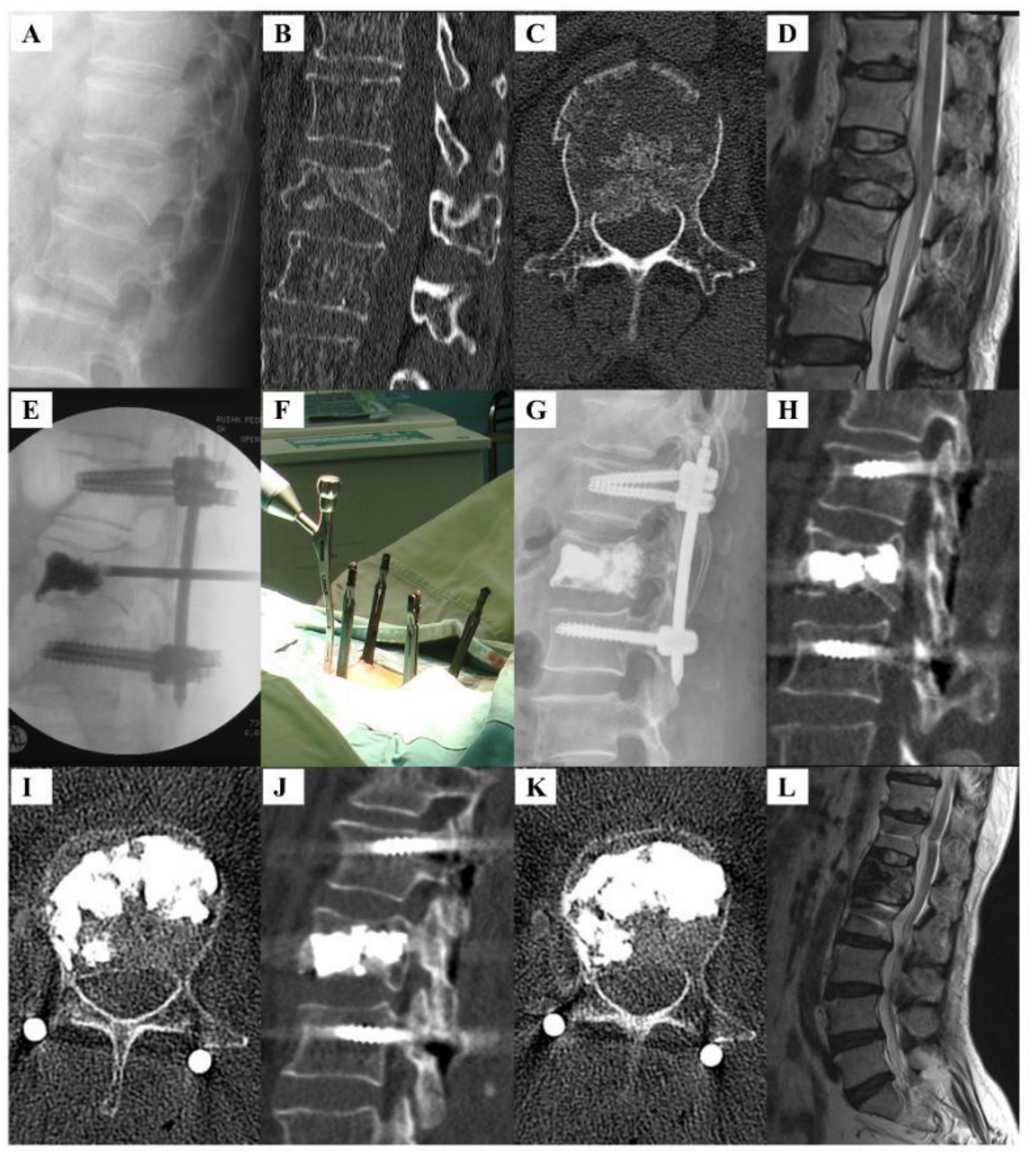

\section{Figure 1}

Female patient of 60 years with OVBF in L1 vertebra undergoing pedicle screw fixation plus vertebroplasty. (A): Preoperative lateral radiograph showing a burst fracture of L1. (B, C): Preoperative CTscan (plain and sagittal reconstruction image) showing the burst fracture with spinal canal compromise. (D): Preoperative MRI (T2-weighted sequences) showing the burst fracture with spinal canal compromise. (E):Intraoperative fluoroscopic image demonstrating percutaneous pedicle screws in the adjacent 
vertebrae and vertebroplasty in the fractured vertebra. (F): Intraoperative view. (G): Postoperative lateral roentgenogram showing adequate vertebral body reduction, excellent alignment and reduced spinal canal encroachment following short fixation and adequate vertebral body reduction. $(\mathrm{H}, \mathrm{I})$ : CT-scan (plain and sagittal reconstruction image) showing adequate vertebral body reduction, excellent alignment and reduced spinal canal encroachment following short fixation and adequate vertebral body reduction. $(J, K)$ CT-scan (plain and sagittal reconstruction image) of the patient one year after surgery showing vertebral body reduction, alignment and reduced spinal canal encroachment without significant loss of correction. (L): T2-weighted sagittal MRI of the patient 4 years after surgery showing reduced spinal canal encroachment and excellent alignment and adequate vertebral body reduction. 


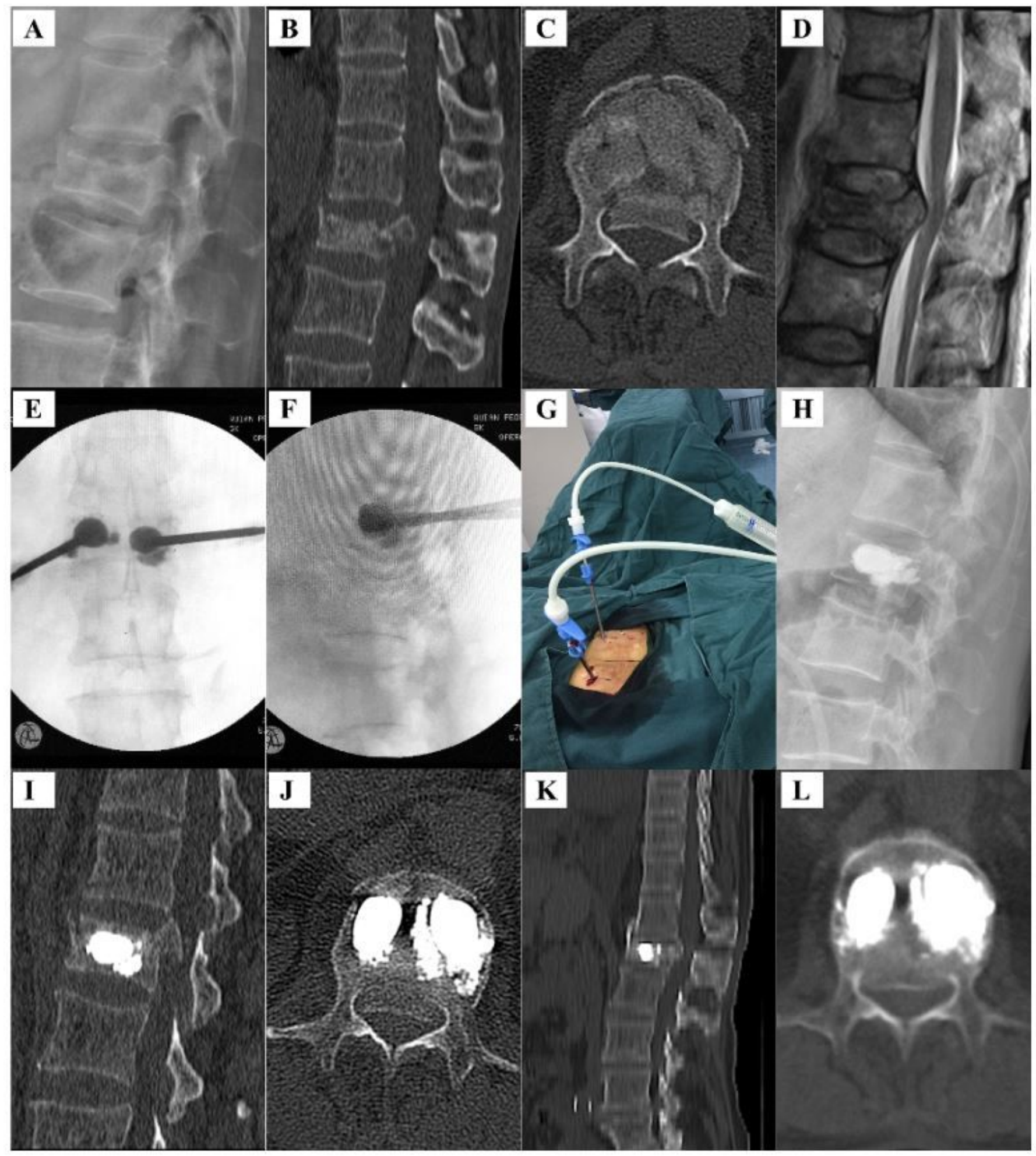

Figure 2

PMCP surgical procedure for the treatment of a 65-year-old female patient with OVBF in L1 vertebra. (A): Preoperative lateral radiograph showing a burst fracture of L1. (B, C): Preoperative CT-scan (plain and sagittal reconstruction image) showing the burst fracture with spinal canal compromise. (D): Preoperative MRI (T2-weighted sequences) showing the burst fracture with spinal canal compromise. (E, F): Intraoperative fluoroscopic image demonstrating PMCP surgical procedure. (G): Intraoperative view. 
(H): Postoperative lateral radiograph showing better alignment following cement injection and adequate vertebral body reduction. $(\mathrm{I}, \mathrm{J})$ : Postoperative CT-scan (plain and sagittal reconstruction image) showing no worse spinal canal compromise and better alignment and adequate vertebral body reduction. $(K, L)$ : CT-scan (plain and sagittal reconstruction image) two years after surgery showing reduced spinal canal compromise, excellent fracture healing and alignment. 